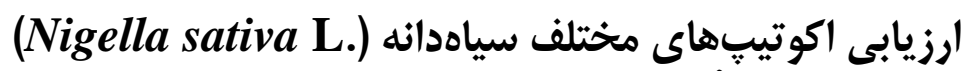 تحت تأثير تنش خشكى دخى در مرحلدى كلدهى
}

\author{
حسن سردارى '، رسول اصغرى زكريا؟، ناصر زارع بّ، ليلا غفارزاده نمازى و و مينا مقدسز ادهْ

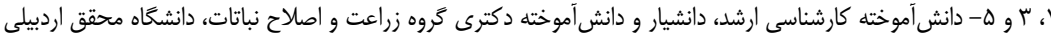

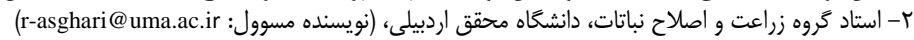

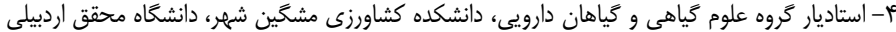 \\ تاريخ دريافت:

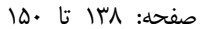

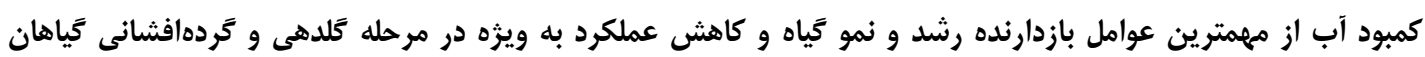

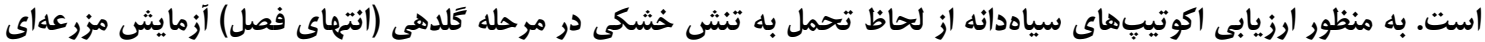

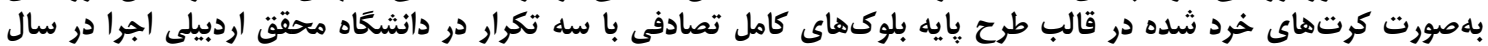

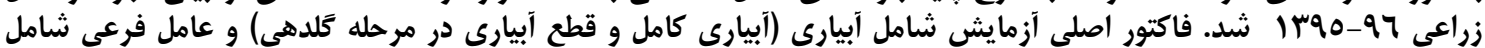

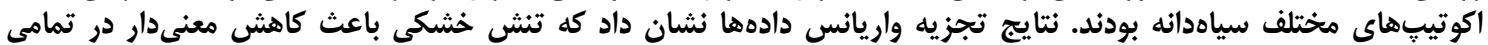

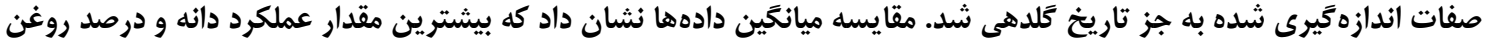

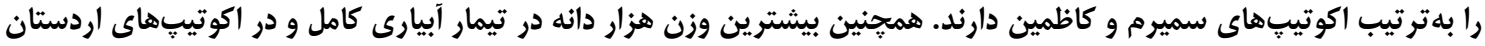

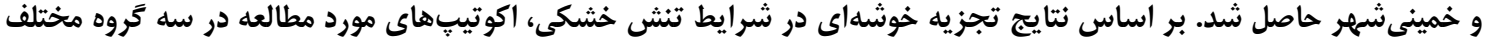

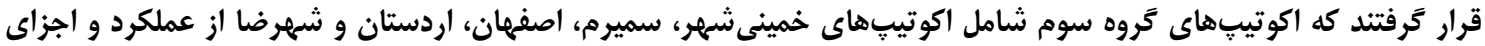

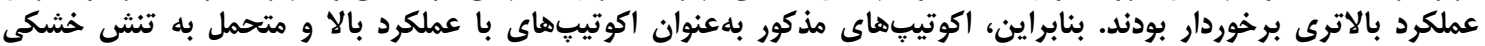

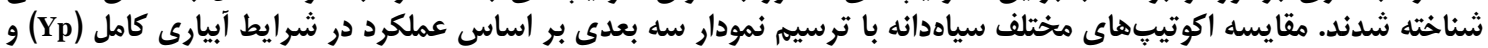

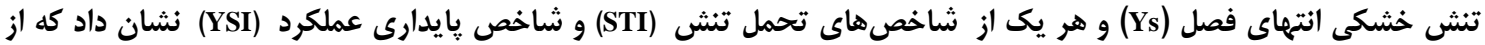

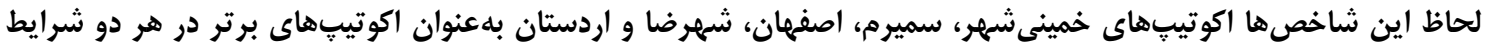
محسوب مى لشوند.

وازههاى كليدى: تجزيه خوشهاى، تنش خشكى، شاخص تحمل تنش، سياهدانه، عملكرد دانه

براى كاهش درد و عوارض ناشى از زَش مار و عقرب كاربر داري

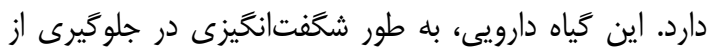

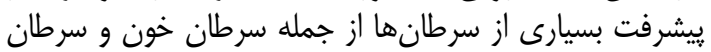

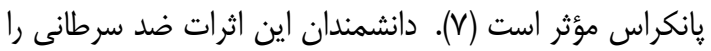

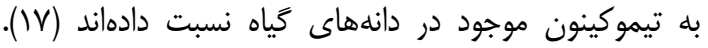

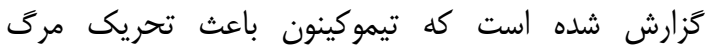

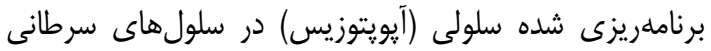

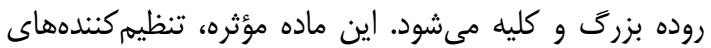

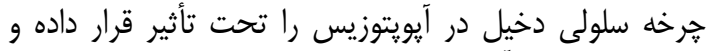

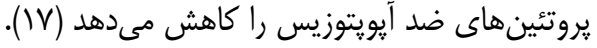

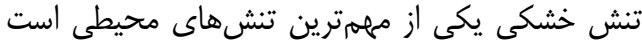

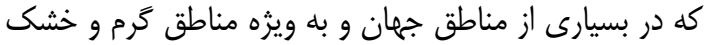

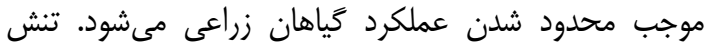

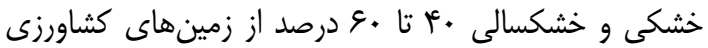

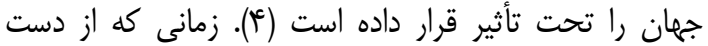

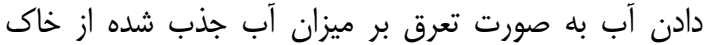

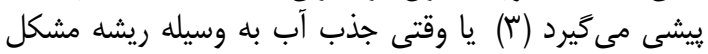

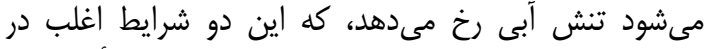

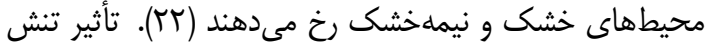

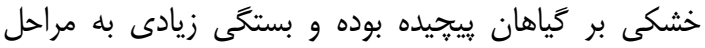

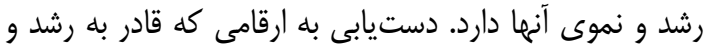

سياهدانه (Nigella sativa L.) كياهى دييلوئيد با عدد

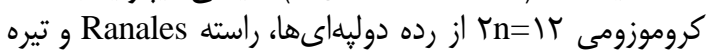

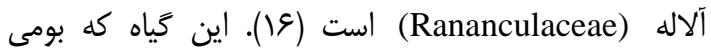

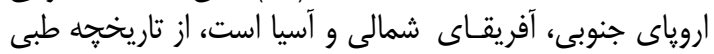

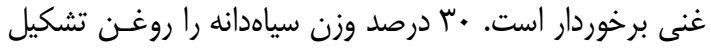

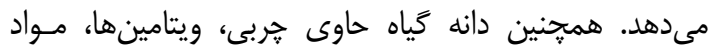

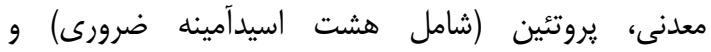

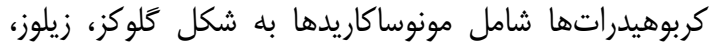

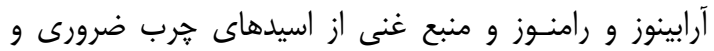

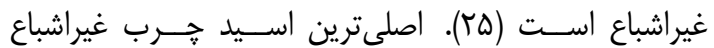

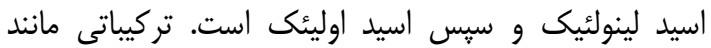

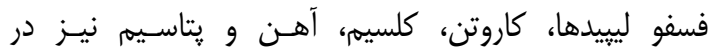

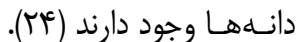

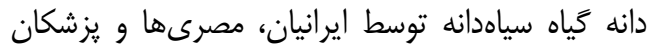

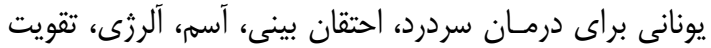

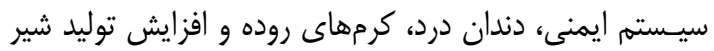

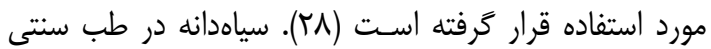

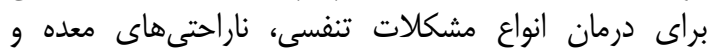

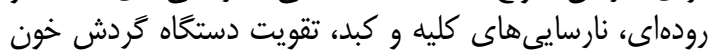

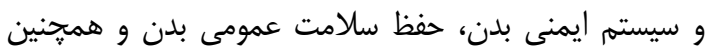




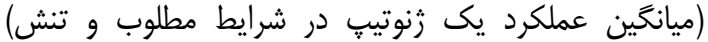

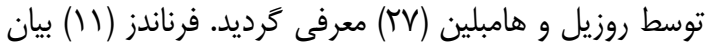

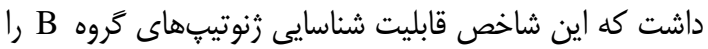

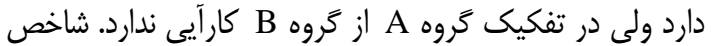
توسط روزيل و هامبلين (TOL

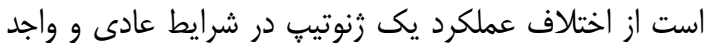

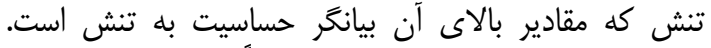

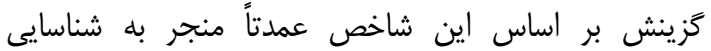

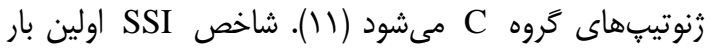

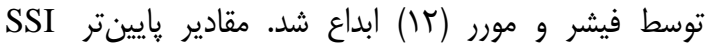

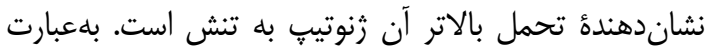

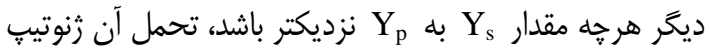

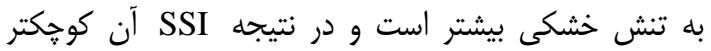

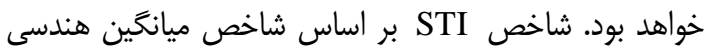

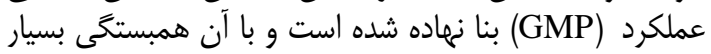

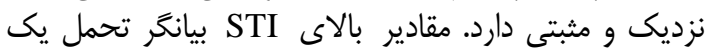

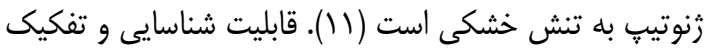

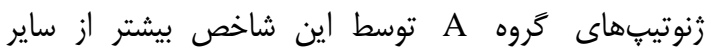
شاخصها است (11). با توجه به وجود تنش خشكى آنى آخر فصل در اكثر آثر نقاط

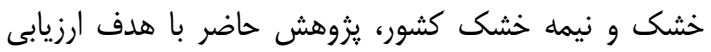

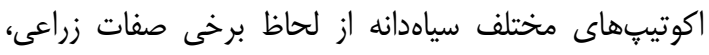

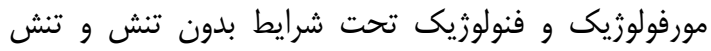

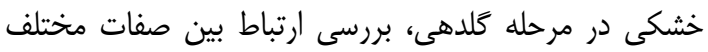

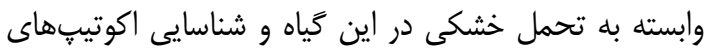

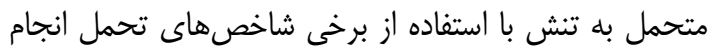
كرفت.

\section{مواد و روشها}

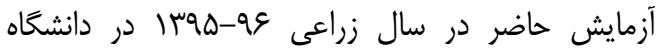

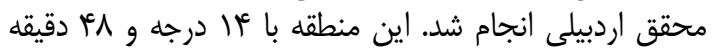

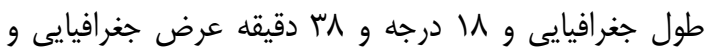

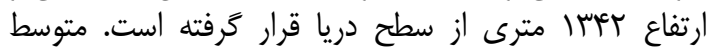

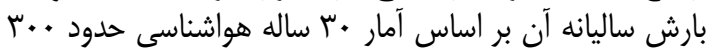

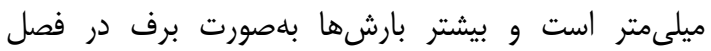

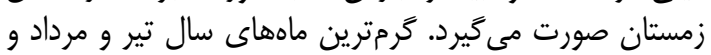

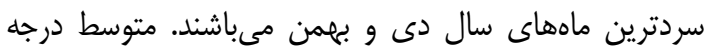

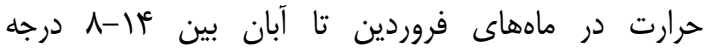

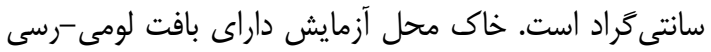
بود. برخى از خصوصيات فيزيكى و استى شيميايى خاك إن محل آزمايش در جدول ا ذذر شده است.
نمو و توليد محصول بالا در شرايط تنش خشكى باشند بسيار

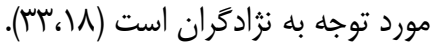

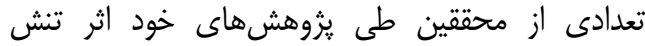

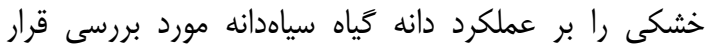

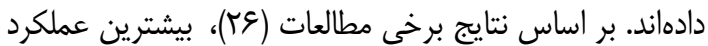

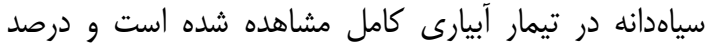

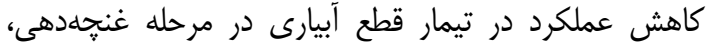

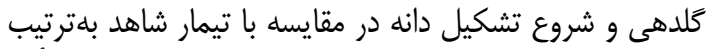

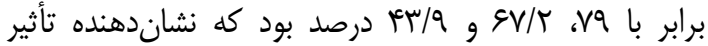

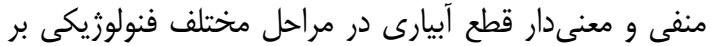

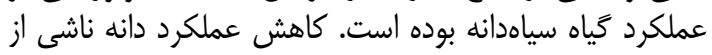

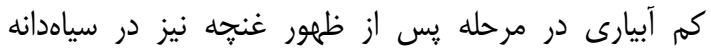

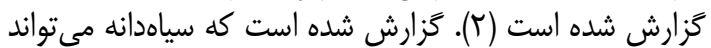

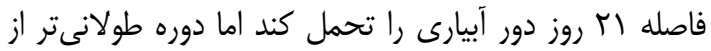

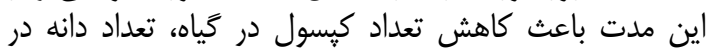

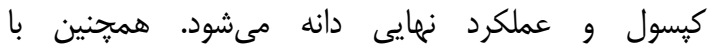

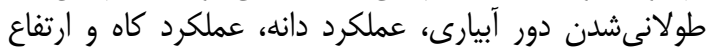

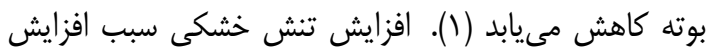

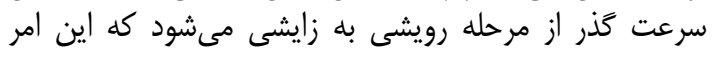

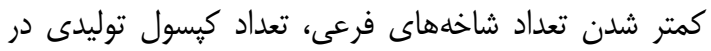

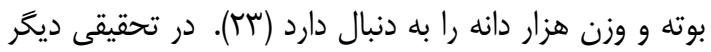
ارزيايى دو گونه No sativa

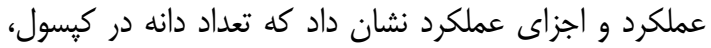

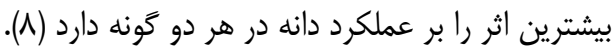

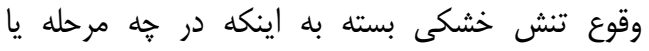

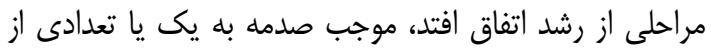

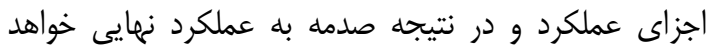

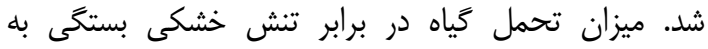

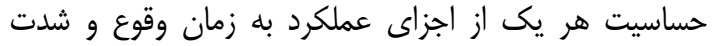

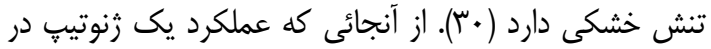

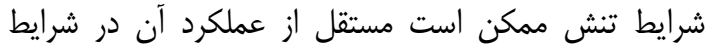

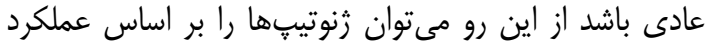

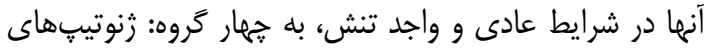

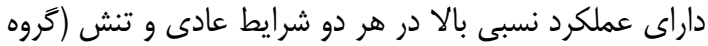

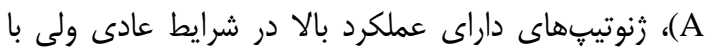

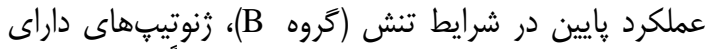

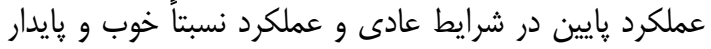

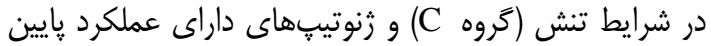

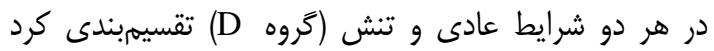

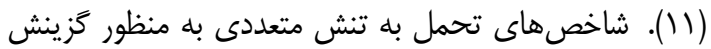

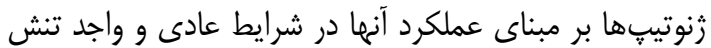

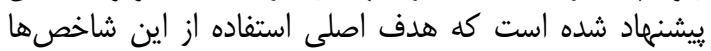

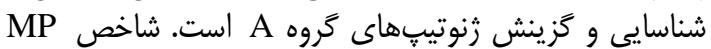


Table 1. Chemical and physical analysis of soil of the experimental site

$$
\text { جدول ا- آناليز شيميايى و فيزيكى خاك محل آزمايش }
$$

\begin{tabular}{|c|c|c|c|c|c|c|c|c|}
\hline هدايت الكتريكى (EC) (1) & $\begin{array}{c}\text { اسيديته } \\
\text { pH }\end{array}$ & $\begin{array}{l}\text { نيتروزن } \\
\text { (ppm) }\end{array}$ & $\begin{array}{c}\text { فسف) } \\
\text { (ppm) }\end{array}$ & يتاسهم & $\begin{array}{l}\text { سيلت } \\
\text { (\%) }\end{array}$ & $\begin{array}{l}\text { ر) } \\
\text { (\%) }\end{array}$ & ش) & بافت خاى \\
\hline.$/ 8 \wedge Y$ & V/AT & 9. & $\mathrm{~V} / \cdot \mathrm{V}$ & Irg & 19 & Tr & tr & لومى رسى \\
\hline
\end{tabular}

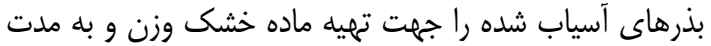

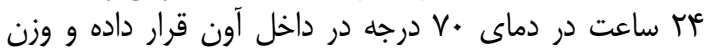

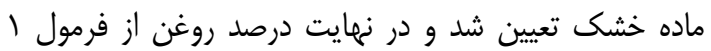
محاسبه گر مديد:

$$
\text { = b-c/b-a ×100 }
$$

كه در آن a وز a كاغن كاغذ صافى بدون نمونه، b وزن كاغذ

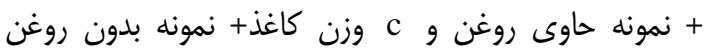

$$
\text { بودند. }
$$

به منظور ارزيابى اكوتيڤها از لحاظ تحمل به خشكى، از (از

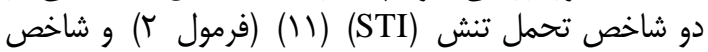

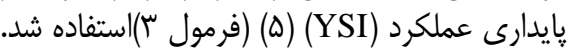
شاخص تحمل تنش (STI) (فرمل (ST) (فرمل r)

$$
S T I=\left(\frac{Y_{p}}{\bar{Y}_{p}}\right)\left(\frac{Y_{s}}{\bar{Y}_{s}}\right)\left(\frac{\bar{Y}_{s}}{\bar{Y}_{p}}\right)=\frac{\left(Y_{p}\right)\left(Y_{s}\right)}{\left(\bar{Y}_{p}\right)^{2}}
$$

شاخص پايدارى عملكرد (YSI) (فرمول r):

$$
\mathrm{YSI}=\frac{\mathrm{Y}_{\mathrm{s}}}{\mathrm{Y}_{\mathrm{p}}}
$$

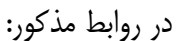

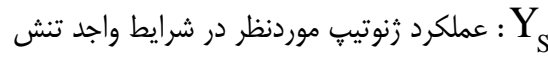

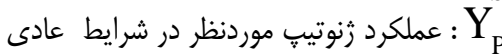
متوسط عملكرد كليه زنوتيبها در شرايط واجد تنش : متوسط عملكرد كليه زنوتيبها در شرايط عادى :

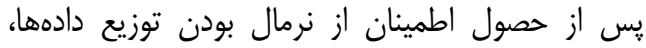

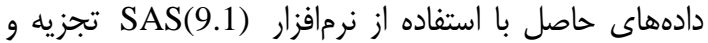

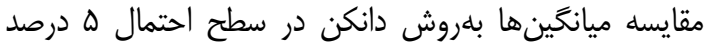

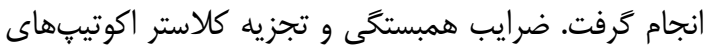

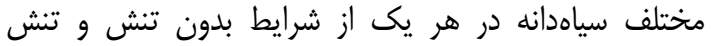

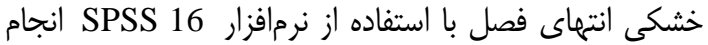
شد. نمايش اكوتيتها بر اساس شاخص انتهاى

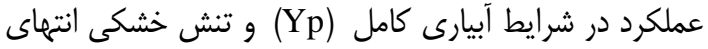

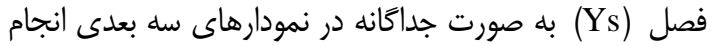

\section{نتايج و بحث}

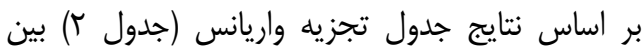

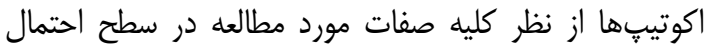

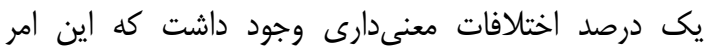

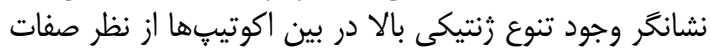

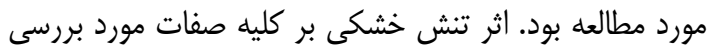

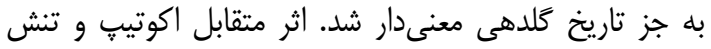

آزمايش بلهورت كرتهاى خرد شده در قالب طرح پايه

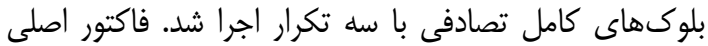

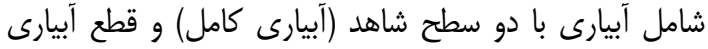

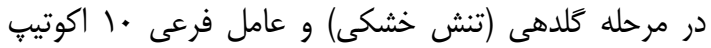

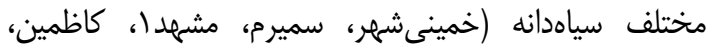

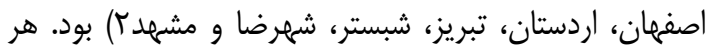

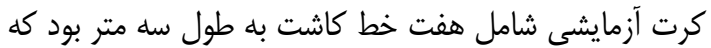

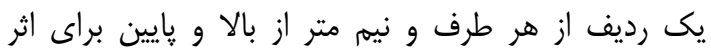

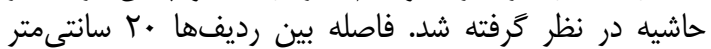

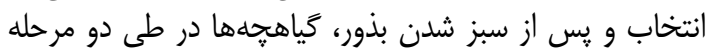

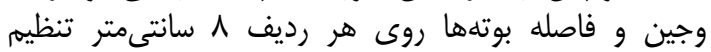
گرديد. دور آبيارى بر اساس نياز ظاهرى گياه

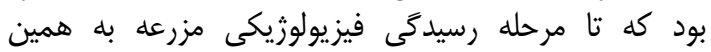

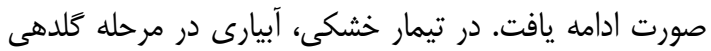

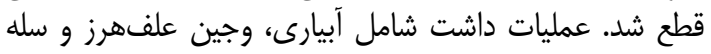

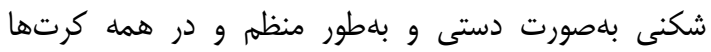

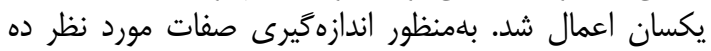

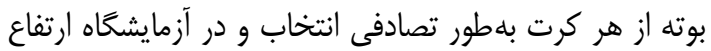

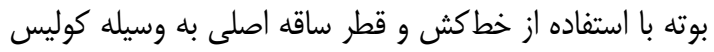

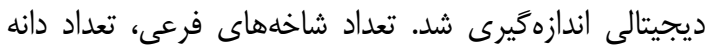

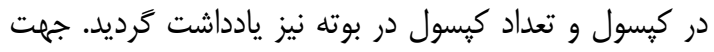

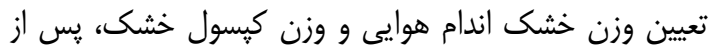

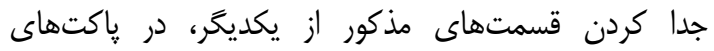

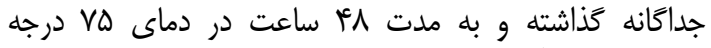

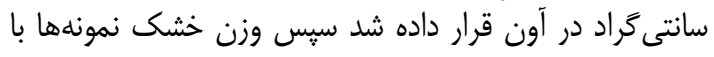

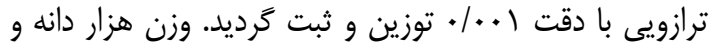

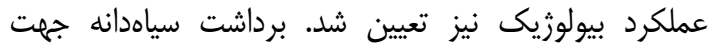

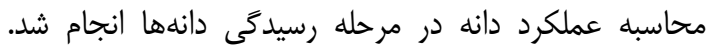

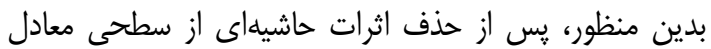

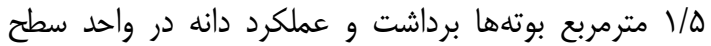

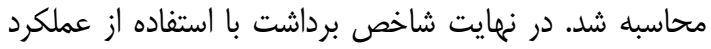
دانه و عملكرد بيولوزيك محاسبه ثر درديد. اندازه كيرى درصد برد روغن با استفاده از دستخاه سوكسله

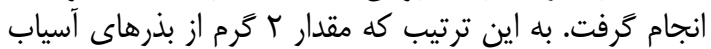

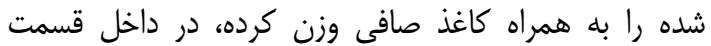

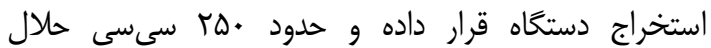

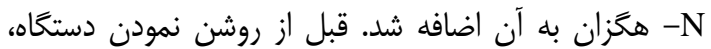

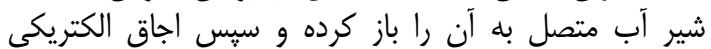

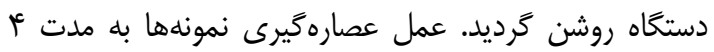

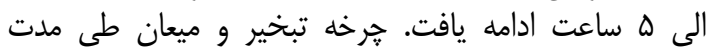

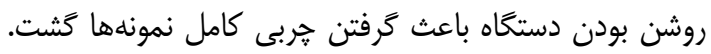

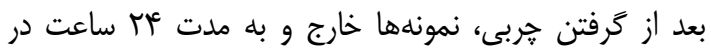

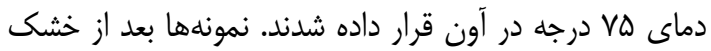

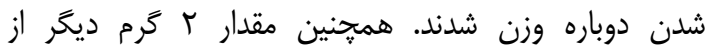


ذكر شده است. تنش خشكى موجب كاهش معنى دار ميانگين

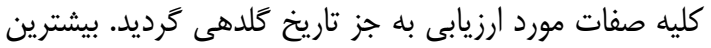
درصد كاهش مربوط به صفت تعداد دانه در كيسول بود.
خشكى بر ارتفاع بوته، وزن خشك بوته، تعداد دانه در كِيسول،

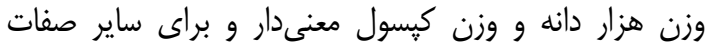

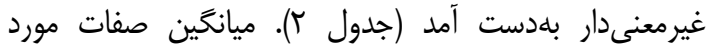
مطالعه در دو شرايط آبيارى كامل و تنش خدس خدكى در جدرل

جدول r- تجزيه واريانس براى صفات مختلف در اكوتيبهاى سياهدانه در شرايط آبيارى كامل و تنش خشكى Table 2. Analysis of variance for various traits in black cumin ecotypes under complete irrigation and end season drought stress conditions

\begin{tabular}{|c|c|c|c|c|c|c|c|}
\hline \multicolumn{6}{|c|}{ ميانكين مربعات } & \multirow[b]{2}{*}{ درجه آزادى } & \multirow[b]{2}{*}{ منبع تغييرات } \\
\hline ارتفاع بوته & تعداد شاخه فرعى & تعداد دانه در & تعداد كيسول & وزان هزار & عملكرد دانه & & \\
\hline .1 .9 & $F \Delta / .9$ & $11 / \cdot 0$ & (צ' & .1 .19 & $G \mid / r)$ & $r$ & تكرار \\
\hline$\Delta \cdot c / \xi .^{* *}$ & $\Delta \wedge १ / q^{* *}$ & ץ../r/" & $F T / A r^{* * *}$ & $\cdot / V \Delta^{*}$ & $|\wedge q| / 9 q^{* *}$ & 1 & تنش خشكى \\
\hline$\cdot / \Lambda$ & $1 . / 19$ & $1 . / M$ &.$/ 98$ &.$/ \mu$ & $N / 4$. & r & خطاى اصلى \\
\hline $\mid W V \% / *^{* *}$ & $\mid$ rqฬ/g.** & $\mu \cdot V / \propto q^{* *}$ & $\mathrm{~V} / \mathrm{V} \mathrm{r}^{* *}$ & $\cdot / r *^{* *}$ & ATFY/DI ${ }^{* *}$ & 9 & اكوتيبٍ \\
\hline$r \mid / r^{* *}$ & $r \cdot v / q{ }^{n s}$ & $\mu F / \mu \cdot *$ & $1 / 1 n^{n s}$ & $\cdot / \cdot e^{*}$ & $|f \cdot| q \Lambda^{\mathrm{ns}}$ & 9 & تنش خشكى × اكوتيبٍ \\
\hline$r / \mathrm{r}$ & $\mid Q N / 9$. & $\mid F / A V$ & $1 / 10$ & $.1 \cdot r$ & $1 \ldots / 41$ & rq & خطاى فرعى \\
\hline$r / 99$ & rY/s. & N/q. & $8 / 99$ & $\Delta / 4 q$ & 1./r & & ضريب تغييرات (درصد) \\
\hline
\end{tabular}

Table 2. Continued

ادامهى جدول r

\begin{tabular}{|c|c|c|c|c|c|c|c|c|}
\hline \multicolumn{7}{|c|}{ ميانگين مربعات } & \multirow[b]{2}{*}{ آزادى درجه } & \multirow[b]{2}{*}{ منبع تغييرات } \\
\hline درصد روغن & وزن خشك & برداشت & قطر ساقه & وزن خيسول خشك & تاريخ رسيدگى & تاريخ كلدهى & & \\
\hline $9 / 1 \pi$ & $\cdot / \cdot 1$ & $19 / 9 \Lambda$ & $\cdot / 4 r$ & $.1 \cdots 9$ & $9 / \Gamma 1$ & $T \cdot / T)$ & $r$ & تكرار \\
\hline VN/RT" & $1 / A r \Lambda^{n-\cdots}$ & $\varepsilon \Delta / r \omega^{n \cdots}$ & $r / q^{n+m}$ & $\cdot / \cdot 1 \cdot r^{n-m}$ & $\mid Q F=/ K g^{n+m}$ & $r / r \varepsilon^{\mathrm{ns}}$ & 1 & تنش خشكى \\
\hline$r q / 10$ & $\cdot / r \Lambda$ & $\Delta / M$ & (ז/. &.$/ \cdots 1$ & $\| T / \Delta \mid$ & $|r / \mu|$ & r & خطاى اصلى \\
\hline$\Delta q / \wedge q^{\cdots *}$ & $\mid F / \cdot 9^{m-m}$ & 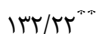 & $. \mid 0)^{m-n}$ & $\cdot / \cdots r^{w n}$ & 更/ & $r \cdot q / k \cdot \cdots$ & 9 & اكوتيب \\
\hline $1 \cdot /{ }^{\mathrm{ns}}$ & $.1 .9^{\prime \prime}$ & $11 / \kappa e^{\mathrm{ns}}$ & $\cdot 1 \cdot 9^{\text {ns }}$ & $\cdot / \cdots r+m$ & $r y / 1^{n s}$ & $9 /{ }^{\mathrm{nss}}$ & 9 & تنش خشكى × اكوتيب \\
\hline $\mid N / \Delta \Lambda$ &.$/ \cdot c$ & $11 / \cdot r$ &.$/ 1 r$ & $\cdot / \cdots \cdot \wedge$ & ID/AT & rI/vg & ع ( ) & خطاى فرعى \\
\hline$|r / 9|$ & $r / \mu 1$ & $15 / 19$ & $\mid r / 9 \Lambda$ & $1 / 4 T^{4}$ & T/Tr & S/AT & & ضريب تغييرات (درصد) \\
\hline
\end{tabular}

جدول ب- تغيير در ميانخين صفات مختلف اكوتيبهاى سياهدانه در شرايط آبيارى كامل و تنش خشكى Table 3. Change in mean of various traits of black cumin ecotypes under complete irrigation and drought stress

\begin{tabular}{|c|c|c|c|c|c|c|c|}
\hline درصد روغن & شاخص برداشت & (قطر ساقه & تعداد شاخه & تعداد كيسول در & 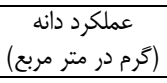 & 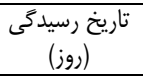 & سر ايط ل ت \\
\hline$r r / / r^{a}$ & "سr/אז & $r / 9 q^{a}$ & $\Delta \cdot / \Lambda \cdot^{a}$ & $\mid V / \mu^{a} \Lambda^{a}$ & $1 \cdot r / V)^{\mathrm{a}}$ & $\mid r F / \Lambda \mu^{a}$ & أبيارى كامل \\
\hline$r q / \Lambda r^{D}$ & TF/ & $T / F \theta^{D}$ & $\Gamma \varphi / \Delta \mu^{D}$ & $10 / 89^{\circ}$ & $91 / \wedge^{\circ} \Lambda^{D}$ & $11 \% / V \cdot^{0}$ & تنش خشكى \\
\hline $\mathrm{V} / \mathrm{l}$. & $N / \cdot 1$ & $\mid \mathrm{N} / \cdot \mathrm{9}$ & $I T / M Y$ & $9 / V$ & IT/AF & $N / N$ & عد كاهش \\
\hline
\end{tabular}

قبلى روند نزولى تعداد شاخههاى جانبى سياهدانه در سطوح

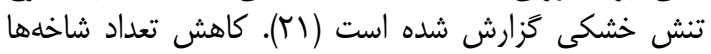

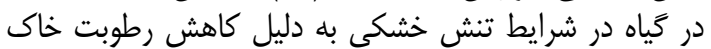

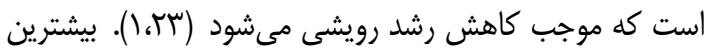

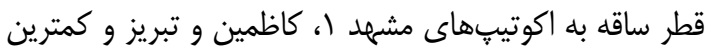

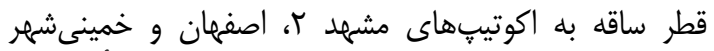

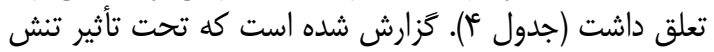

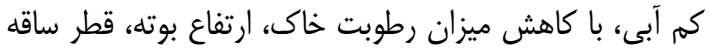

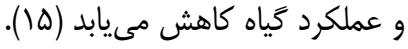

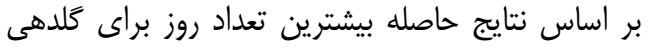

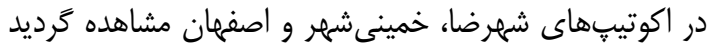

مقايسه ميانگين بين اكوتييٍهاي سياهدانه

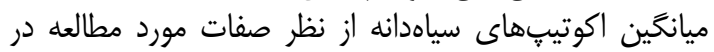

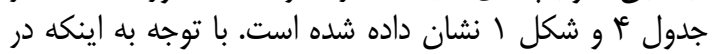

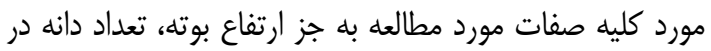

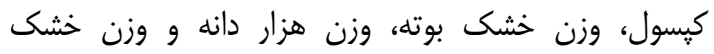

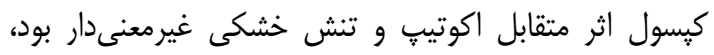

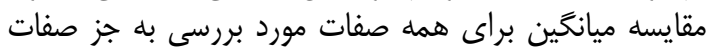

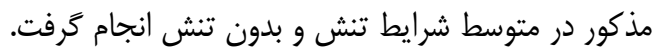

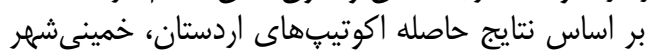

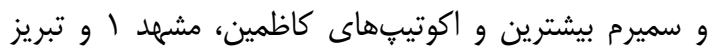
كمترين تعداد شاخه فرعى را داشتند (جدول أل). در تحقيقات 


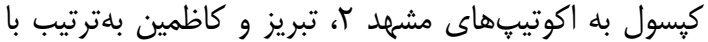

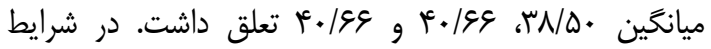

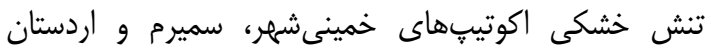

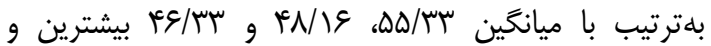

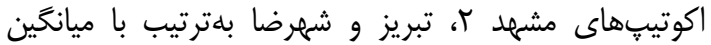

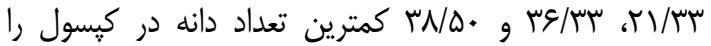

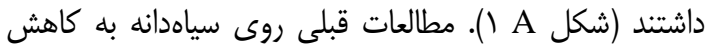

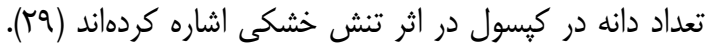

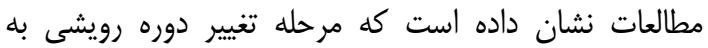

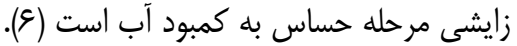

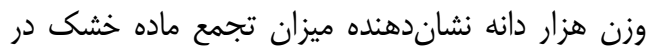

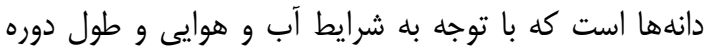

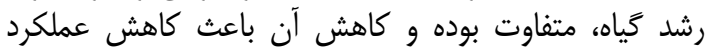

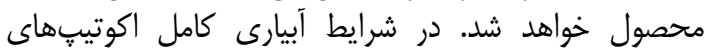

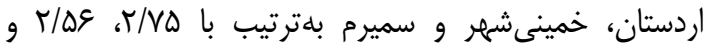

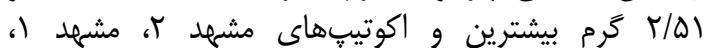

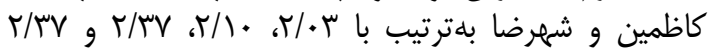

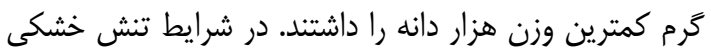

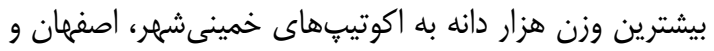

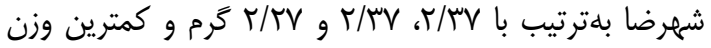

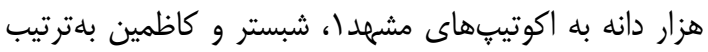

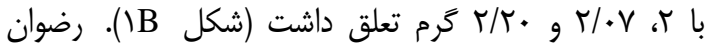

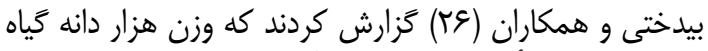

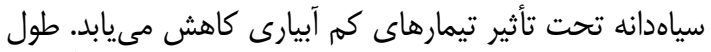

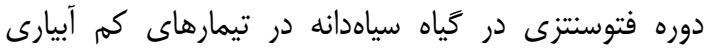

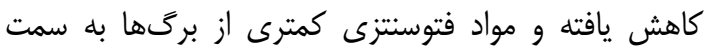

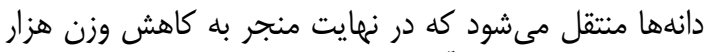

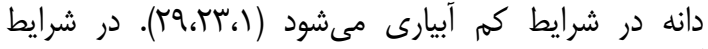

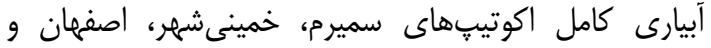

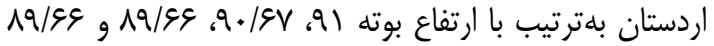

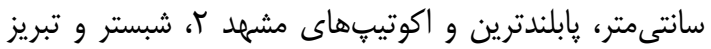

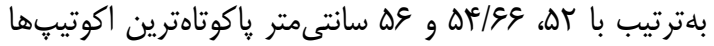

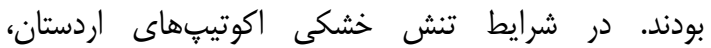

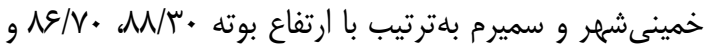

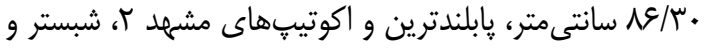

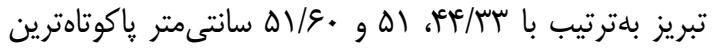

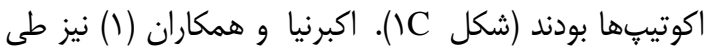

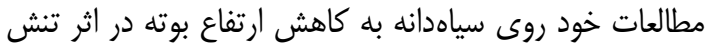

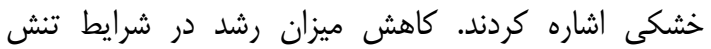

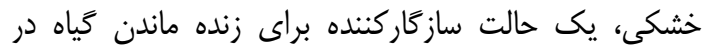

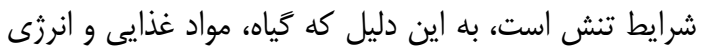

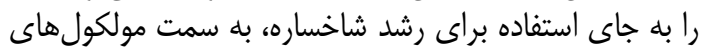

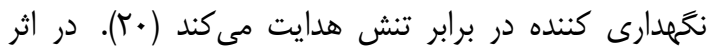
كمبود آب حجم سلول، تقسيم سلولى، ديو ديوارهسازى سلو سلول،

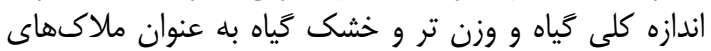

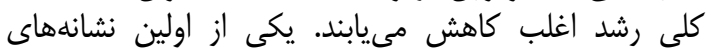

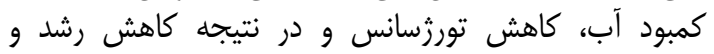

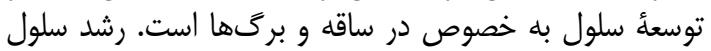
حساسترين فرآيندى است كهو بلهوسيله تنش كهمآبى تحت رلت
و زودترين زمان كلدهى مربوط به اكوتيههاى شبستر، تبريز و

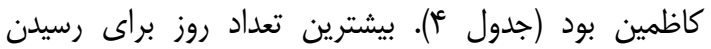

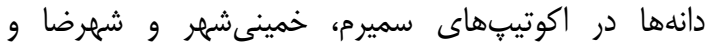

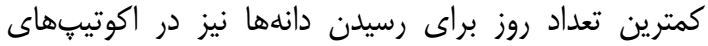

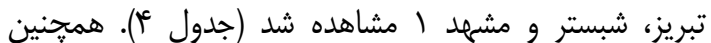

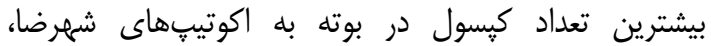

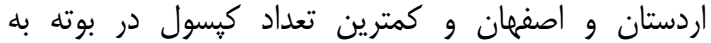

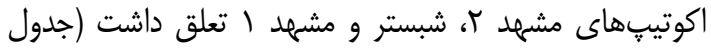

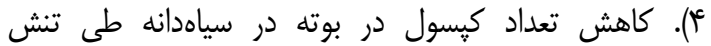

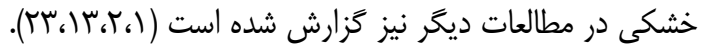

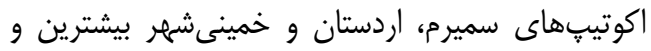

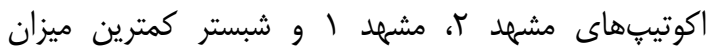

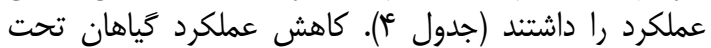

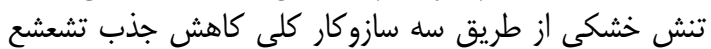
فعال فتوسنتزى، كاهش كارآيى مصرف نوار نور و كاهش داهي در تبادل

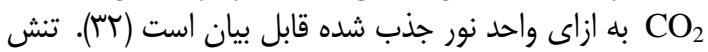

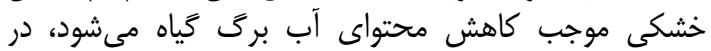

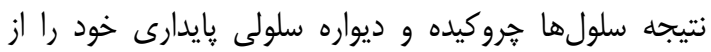

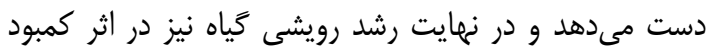

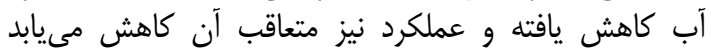

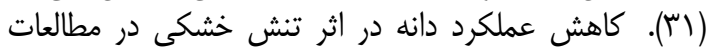

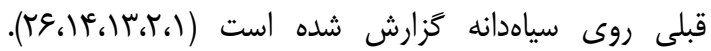

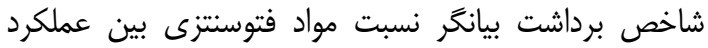

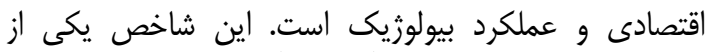

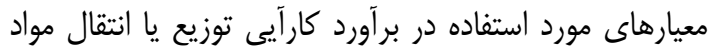

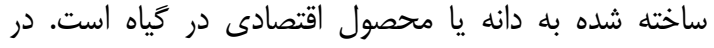

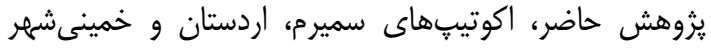

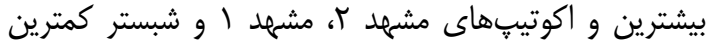

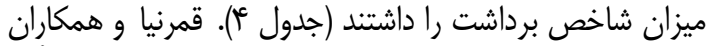

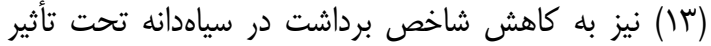
تنش خشكى اشاره كردهاند.

كاهش عملكرد در تيمارهاى تحت تنش تنش آب، ناشى از از افت

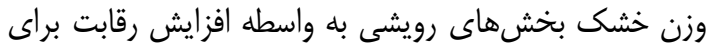

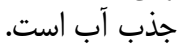

اكوتيههاى كاظمين، تبريز و شبستر بيشترين و و

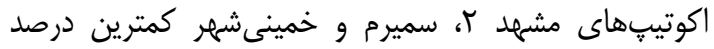

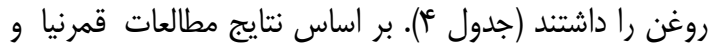

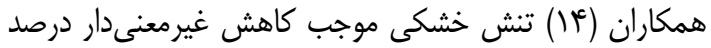

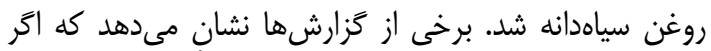

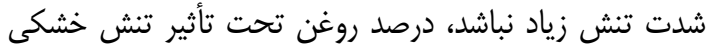

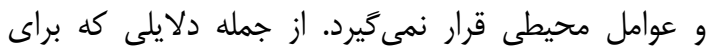

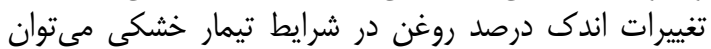

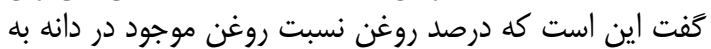

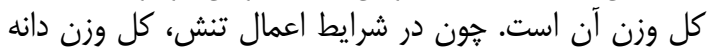

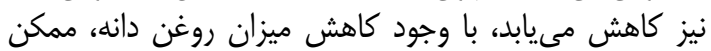

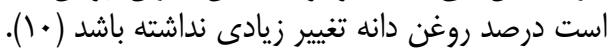

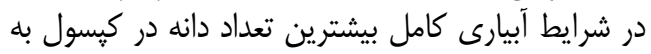

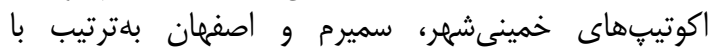

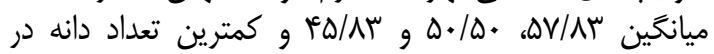




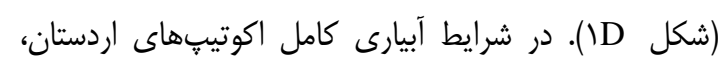

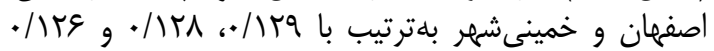

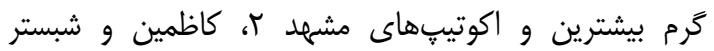

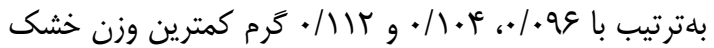

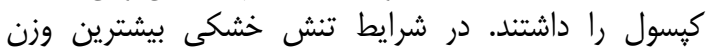

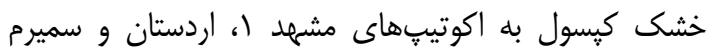

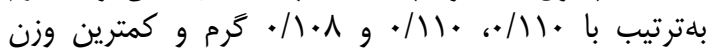

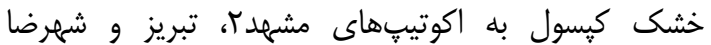

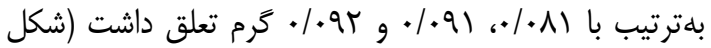

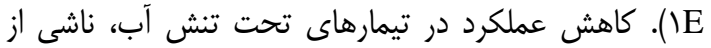

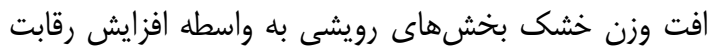
براى جذب آب است.
تأثير قرار مى گيرد. با كاهش رشد سلول اندازه اندام محدود

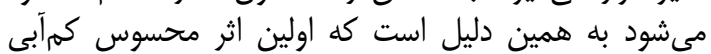

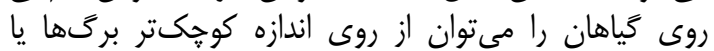

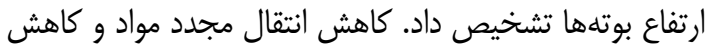

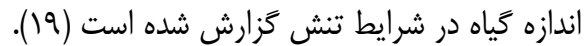

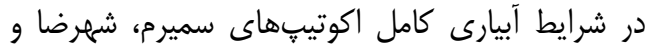

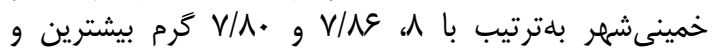

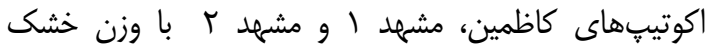

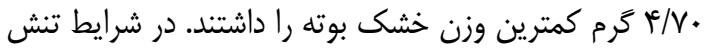

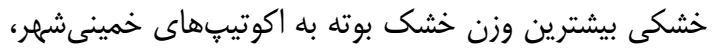

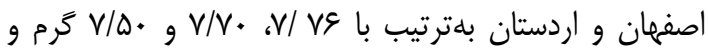

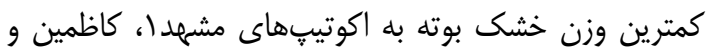

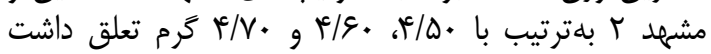

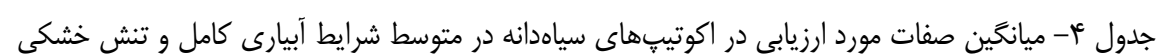
Table 4. Mean of studied traits in black cumin ecotypes in the average complete irrigation and drought stress conditions

\begin{tabular}{|c|c|c|c|c|c|c|c|c|}
\hline درصد روغن & تعداد شاخه & تعدرد كيسول & برداشت & عملكرد دانه & (ميلى متر) & تاريخ رسيدگى & & اكوتيب \\
\hline$r N / \Delta r^{c d}$ & $\Delta \Lambda / \mu^{a}$ & $10 / 9 Y^{c}$ & $r N / 1 q^{a b}$ & (rr/r & $r / 4 q^{c}$ & $\mid r \mu / \Lambda \mu^{a}$ & Khomeini Shahr & خمينىشهر \\
\hline$r V / I V^{d}$ & $\Delta \mathrm{V} / \Delta \cdot^{\mathrm{a}}$ & $19 / / r^{b}$ & $r \cdot / \kappa r^{a}$ & $|f| / 9 \Delta^{a}$ & $r / \Delta \mu^{b c}$ & $\mid r r / g q^{\mathrm{a}}$ & Semirom & سميرم \\
\hline$r \Psi / \mathscr{G} V^{\text {abcd }}$ & $r \Delta / / v^{c}$ & $\mid r / \cdot 1^{e}$ & rM/IfC & $8 \% / 11^{c}$ & $r / r \varepsilon^{a}$ & $\| \Delta / \varphi^{b}$ & Mashhad 1 & مشهدا \\
\hline$r \omega / \omega \cdot \cdot^{a}$ & $r \mathrm{r} / r^{\mathrm{c}}$ & Ir/krde & $r \Delta / \wedge)^{b c}$ & $\checkmark / N^{c}$ & $r / .^{a}$ & $\| 19 / . .^{b}$ & Kazemin & كاظمين \\
\hline$r N / \Lambda r^{c d}$ & b & $19 / 4 \varepsilon^{b}$ & $r \Delta / \Delta \mathrm{V}^{\mathrm{bc}}$ & $\| N / M \Lambda^{b}$ & $T / \kappa^{C}$ & $|r r /| q^{\mathrm{a}}$ & Isfahan & اصفهان \\
\hline$r q / \uparrow \wedge^{b c d}$ & $91 / 19^{\mathrm{a}}$ & $19 / \Delta V^{b}$ & $r \cdot /$ fa & $\left|{ }^{\prime} N /\right|^{a}$ & $r / \Lambda \kappa^{a b c}$ & 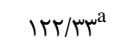 & Ardestan & اردستان \\
\hline$M{ }^{\mu} / \Delta \oint^{\mathrm{ab}}$ & $r N / I V^{c}$ & $\mid F / \xi^{d}$ & $r e / \mu r^{b c}$ & $V \pi / q^{c}$ & $r / q r^{a b}$ & $\| r / \Lambda \mu^{b}$ & Tabriz & تبريز \\
\hline$\mu F / 1 .{ }^{a b c}$ & $\mathrm{FV} / .^{\mathrm{b}}$ & $w / v^{e}$ & $r r / \Delta T^{c}$ & $\mathrm{~V}) / .9^{\mathrm{c}}$ & $r / \mathcal{N} \mathcal{F}^{\mathrm{abc}}$ & b מא/ץ II b & Shabestar & شبستر \\
\hline 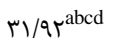 & $\Delta r / \Lambda r^{b}$ & $r I / 9 a^{a}$ & $r \varepsilon / \wedge a^{a b c}$ & $I r \cdot / / v^{b}$ & $T / \Delta F^{b c}$ & 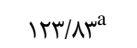 & Shahreza & شهرضا \\
\hline$r V / \cdot r^{d}$ & Fi/Arc & $\mid r / \mu q^{f}$ & $1 \% / 9 \Delta^{\mathrm{d}}$ & $r q / r \cdot{ }^{d}$ & $r / \Gamma^{c}$ & $\mid r / .^{a}$ & Mashhad 2 & مشهدr \\
\hline
\end{tabular}


(A)

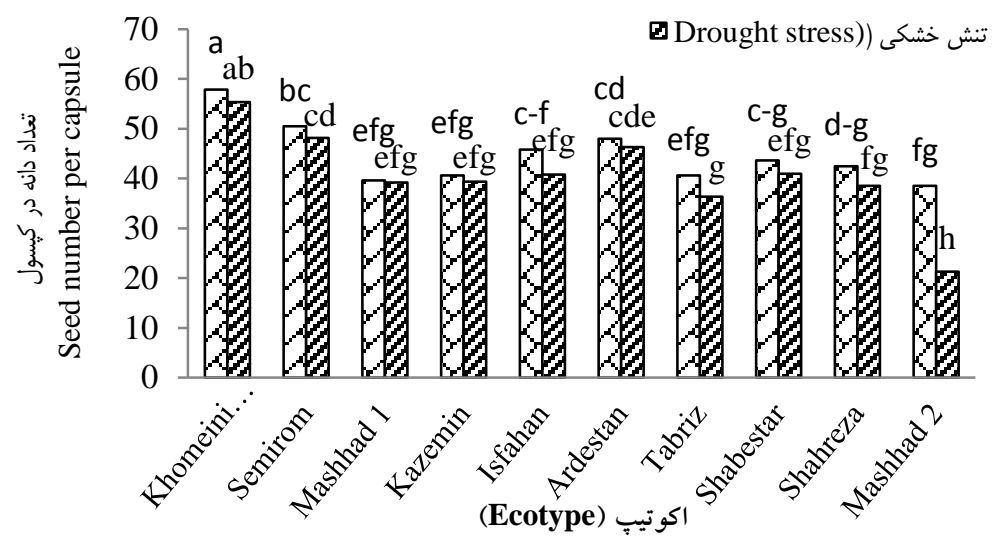

(C)
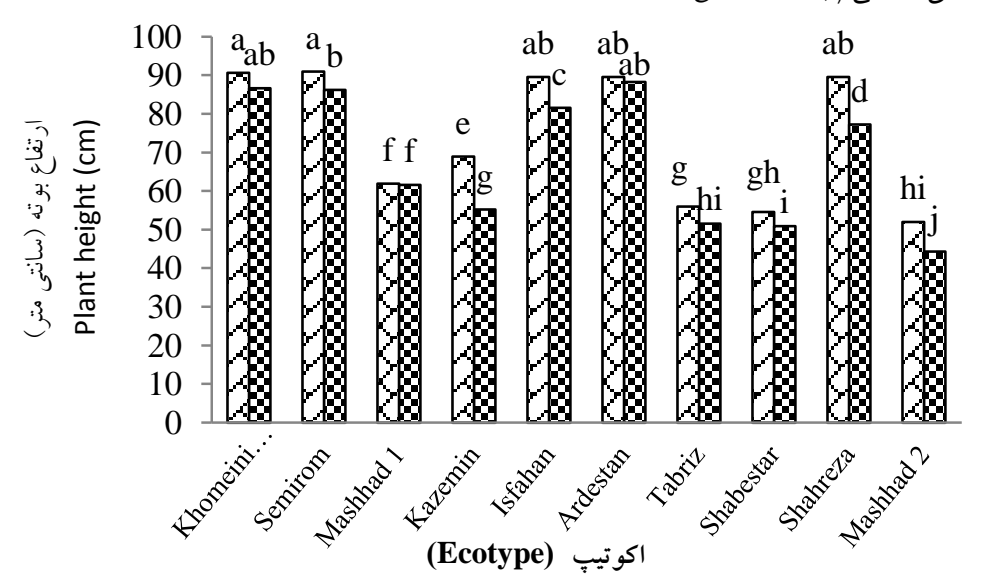

(B) DFull irrigation) (إيارى كامل

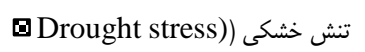
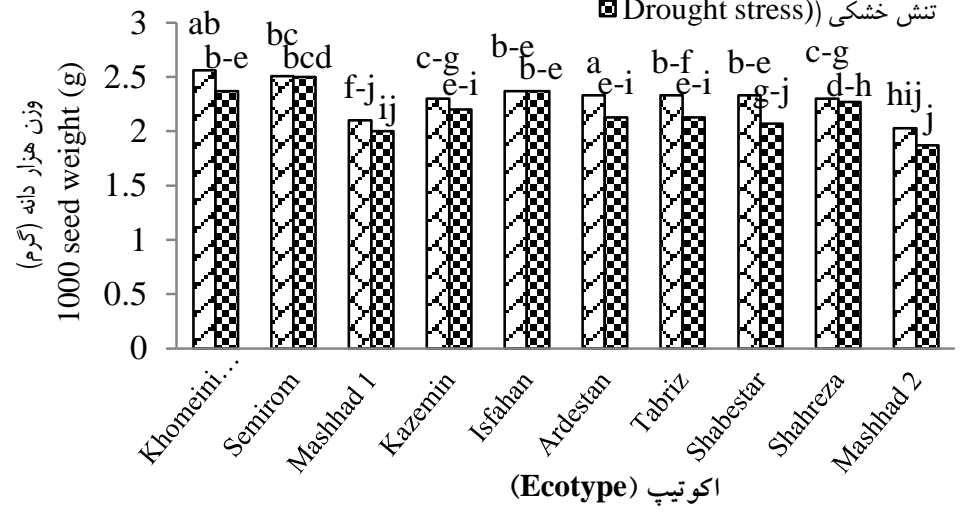

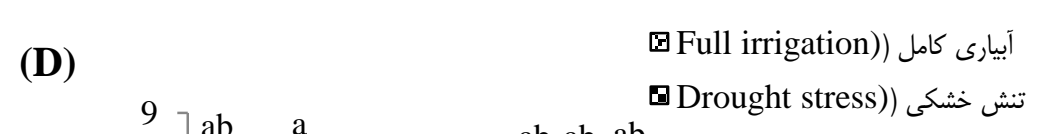

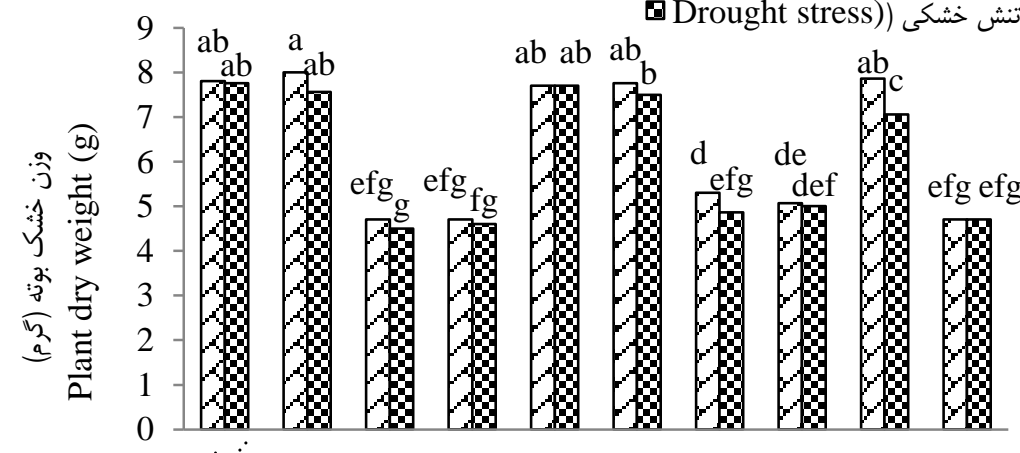

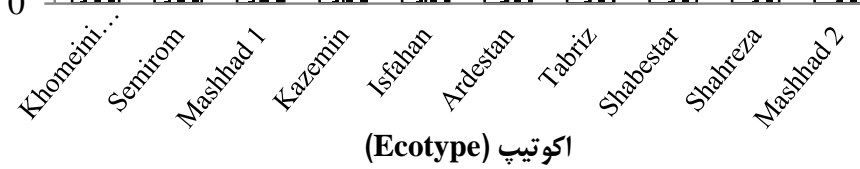

شكل ا- ميانكَين اكوتيبهاى مختلف سياهدانه از لحاظ (A) تعداد دانه در كيسول، (B) وزن هزار دانه، (C) ارتفاع بوته، (D) وزن خشك بوته، و وزن خشى كيسول (E) در شرايط آبيارى كامل و تنش

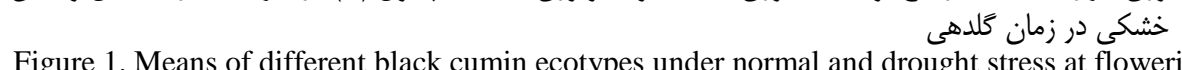

Figure 1. Means of different black cumin ecotypes under normal and drought stress at flowering stage for (A) number of grains per follicle, (B) 1000-grain yield, (C) plant height (D), plant dry weight, and (E) capsule dry weight 
(E)

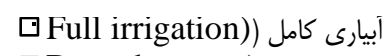

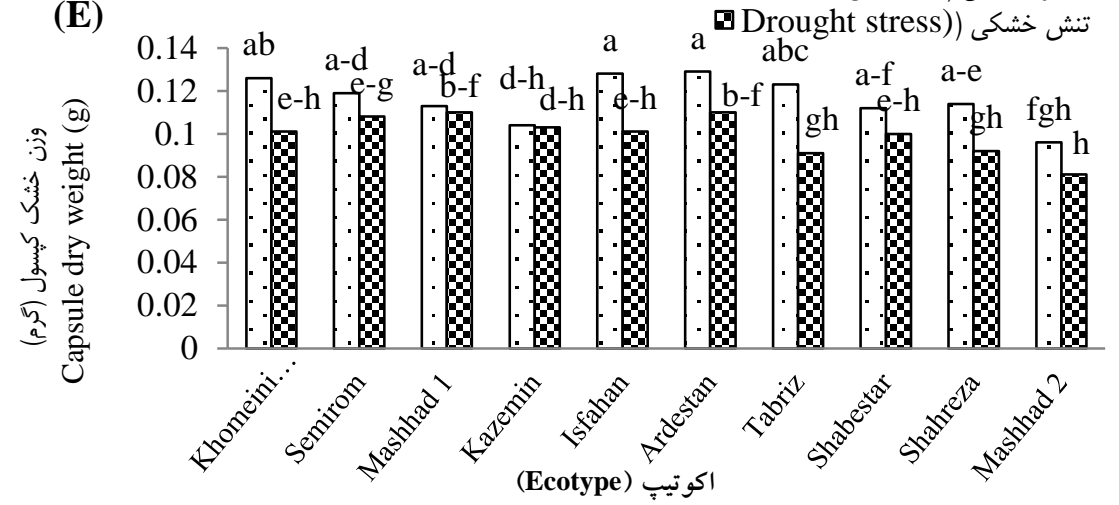

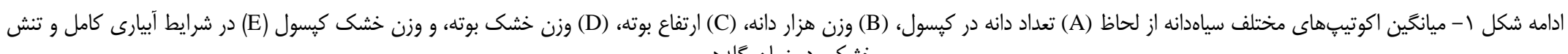
خشكى در زمان كلدهى (Bل

Continued Figure 1. Means of different black cumin ecotypes under normal and drought stress at flowering stage for (A) number of grains per follicle, (B) 1000-grain yield, (C) plant height (D), plant dry weight, and (E) capsule dry weight

جدول ه- ضرايب همبستخى بين صفات مورد ارزيابى در اكوتيِهاى سياهدانه در شرايط آبيارى كامل (اعداد زير قطر) و تنش خشكى (اعداد روى قطر) Table 5. Correlation coefficients among the studied traits in black cumin ecotypes in the full irrigation condition (below the diagonal) and under drought stress condition (on

\begin{tabular}{|c|c|c|c|c|c|c|c|c|c|c|c|c|c|}
\hline قطر ساقه & درصد روغن & 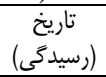 & تاريخ كَلدهى & وزن خشول & شاخص برداشت & عملكرد دانه & وزن خشك & وزن هزار دانه & تعداد كيسول & تعداد دانه در & تعداد شاخه & ارتفاع بوته & صفات مورد ارزيابى \\
\hline$-\cdot / T^{c}$ & $-\cdot / \Delta \Lambda$ &.$/ 41$ &.$/ \Delta \Lambda$ & .10 & $\cdot / A r^{k+\pi}$ & $.199^{\text {sin }}$ &.$/ 99^{201}$ & $\cdot / \Lambda r^{n+m}$ & $\cdot / \Lambda . "$ & $\cdot / \mathrm{VV}$ &.$/ 90$ & $1 / \cdot \cdot$ & ارتفاع بوته (1) \\
\hline$-\cdot / \kappa \Delta$ &.$- / 9 \varphi^{*}$ &.$/ \Delta \Delta$ & $\cdot / N r^{*}$ &.$/ \mu r$ & $\cdot / \mathrm{V} \cdot *$ & $. / 9)^{* * * * *}$ &.$/ 9 V^{* * *}$ & $\cdot / \Lambda^{* * *}$ & $\cdot / 19^{* * *}$ &.$/ 8$ & $1 / \cdot \cdot$ &.$/ 4 \Delta$ & تعداد شاخه فرعى \\
\hline $.1 \cdot 1$ &.$- / R \varepsilon$ &.- .1 .9 & $.1 \cdot 1$ & $\cdot / \Delta \Lambda$ &.$/ 9 * * *$ &.$/ V^{* * * *}$ & $.199^{*}$ & $\cdot / \mathrm{V}^{* * *}$ &.$|4|$ & $1 / \cdot \cdot$ & $\cdot / \Delta V$ & $\cdot / V r^{*}$ & تعداد دانه در كيسول \\
\hline.$- / 4 \Delta$ & r & $\cdot|\Delta|$ & س بع/. & $\cdot 1 \cdot 1$ & $.199^{*}$ & $\cdot / \Lambda \omega^{* *}$ & $\cdot / \Lambda 0^{* *}$ & $\cdot / v^{*}$ & $1 / \cdot \cdot$ &.$/ 4 \Delta$ &.$/ A V$ &.$/ 9 *^{* * *}$ & تعداد كيسول در بوته \\
\hline.$-|4|$ &.$- / 4$ & س (س/. &.$/ 4 T$ & س (س/. &.$/ 9 r^{* * *}$ &.$/ 99^{m * n a n}$ & . $/ \wedge r^{*: *}$ & $1 / \cdot \cdot$ & 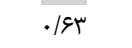 & $\cdot / \mathrm{V} \mathrm{N}^{* * n}$ & $\cdot / V r^{*}$ & $\cdot(V)^{*}$ & وزن هزار دانه \\
\hline$-\cdot / \Delta T$ &.$- / 9 V^{*}$ &.$/ \Delta \Lambda$ & $\cdot / N r^{*}$ & 每 & $\cdot / N e^{*}$ &.$/ 99^{* *}$ & $1 / \cdot \cdot$ & $\cdot / N e^{*}$ & $. / 9 .^{* * * * *}$ & $\cdot / v \Delta^{*}$ & سو/. &.$/ 90^{* * *}$ & وزن خشك بوته \\
\hline.$- / 4 T$ &.$- / \Delta F$ &.$/ \& \Delta$ &.$/ \Delta \Lambda$ & $\cdot / r v$ & $\cdot / 1 q^{* *}$ & $1 / \cdot \cdot$ &.$/ 90^{* * *}$ & $\cdot / M^{* * *}$ & $\cdot / M^{* * *}$ & $\cdot / 1 . * * *$ & $\cdot \mid \& \wedge *$ & $. / 9)^{* * * *}$ & عملكرد دانه \\
\hline.$- / 10$ & $-\cdot / T F$ &.$/ 14$ & $\cdot|r|$ & אזא/. & $1 / \cdot \cdot$ &.$/ 19^{* * * *}$ & $\cdot / v^{*: 3}$ & $\cdot / 9 r^{* * *}$ & $\cdot / V^{*}$ & $\cdot / N e^{* *}$ &.$/ 4 \wedge$ & $\cdot / \Lambda \cdot *$ & شاخص برداشت \\
\hline.$/ 4 \Delta$ &.$- / 1 V$ & $-\cdot / r \Delta$ &.$- / 19$ & $1 / \cdot$ & $.199^{*}$ & $\cdot / \Lambda 0^{* * * n}$ & $\cdot / \mathrm{V}^{* * *}$ & $\cdot / \Lambda r^{* * *}$ & .109 & $\cdot / V q^{*}$ & .190 & $.19 \mathrm{~V}^{*}$ & وزن خشك كيسول \\
\hline-.199 & $-\cdot / v \Lambda^{* *}$ & $.199^{* * *}$ & $1 / \cdot \cdot$ &.$/ 4 \wedge$ & r l l & $.19 \Lambda^{*}$ & $\cdot / \Lambda^{* * * *}$ & &.$/ 9 T$ & $.1 \Delta S$ &.$/ D F$ & $\cdot / \mathrm{V}^{e^{*}}$ & تاريخ كلدهى \\
\hline$-\cdot / v \cdot *$ & $-\cdot / r^{*}$ & $1 / \cdot \cdot$ & $\cdot / 19^{* *}$ & $\cdot / 4 \wedge$ & $\cdot / \Delta \Lambda$ & 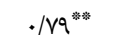 & $\cdot / \mathrm{V} \wedge^{* * *}$ & .109 & $.19 \mathrm{~V}^{*}$ & . &.$/ \Delta \Lambda$ & $\cdot / \Lambda \uparrow^{*: *}$ & تاريخ رسيدگى \\
\hline$\cdot / \Delta V$ & $1 / \cdot$ &.$-|9|$ & $-\cdot / V \Lambda^{* * * *}$ & - & .1 .1 &.$- / \mu r$ &.$- / 4 \wedge$ & $-. \cdot / \cdot v$ &.$- / 49$ & 每/ &.$- / 4$. & g & درصد روغن \\
\hline $1 / \cdot \cdot$ & $\cdot / \Lambda \Delta^{\text {biste }}$ &.$- / 94$ & $-\cdot / \mathrm{M}^{*}$ & $-\cdot / \mu$ & $.1 \cdot 4$ &.$- / \Psi v$ & $-\cdot / \Delta V$ &.$- / \cdot v$ & $-\cdot / \mu$. &.$- / 4 \varphi$ & س &.$- / 49$ & قطر ساقه \\
\hline
\end{tabular}


تجزيه خوشهاى اكوتيبها در شرايط تنش خشكى نيز،

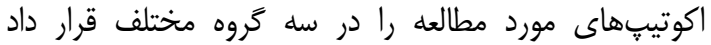

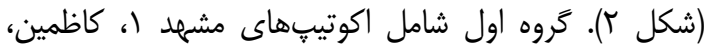

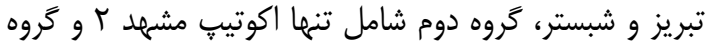

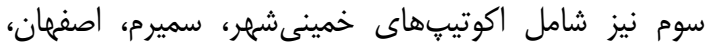

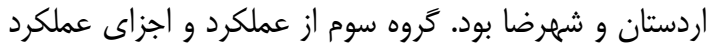

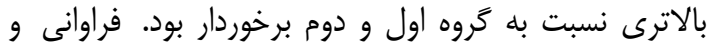

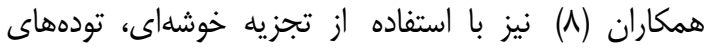

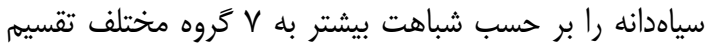

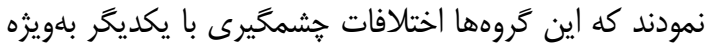
براى صفت ميزان عملكرد داشتند.

مقايسه اكوتيّهاى مختص مختلف سياهدانه بر اساس شاخص هاى تحمل تنش إنش

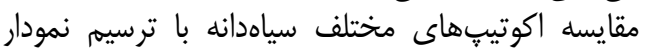

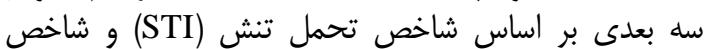

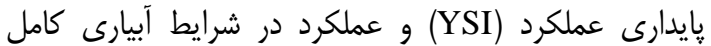

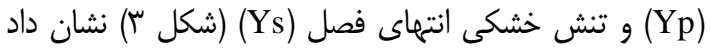

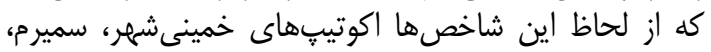

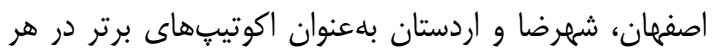

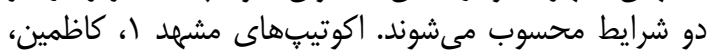

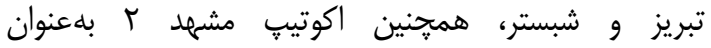

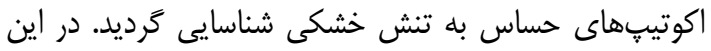

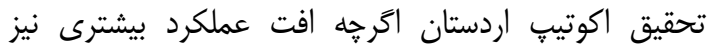

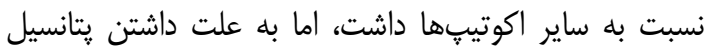

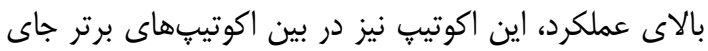

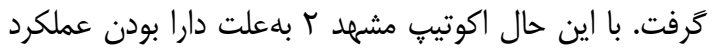

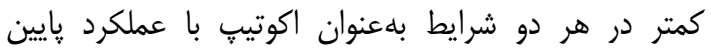

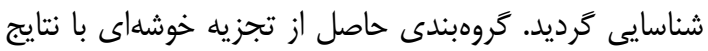

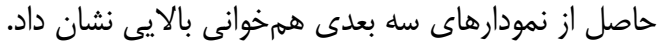

همبستكى بين صفات

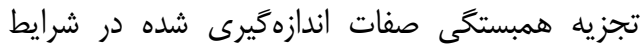

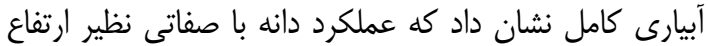

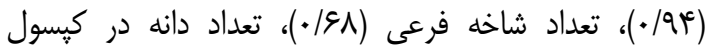

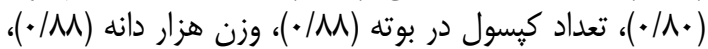

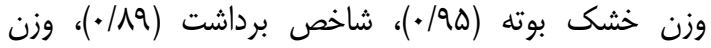

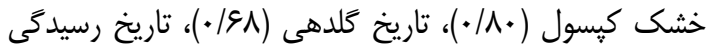

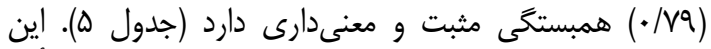

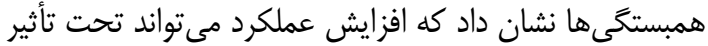

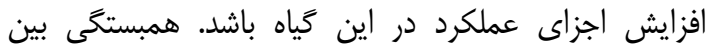

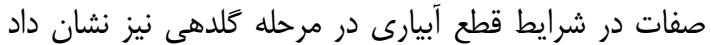

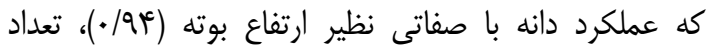

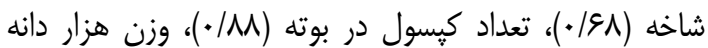

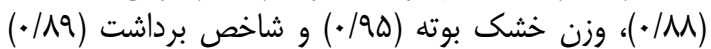

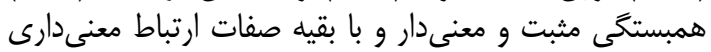

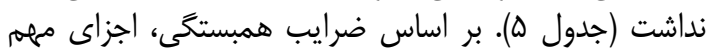

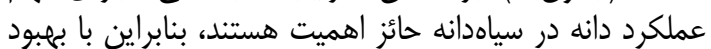

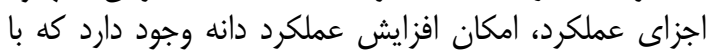

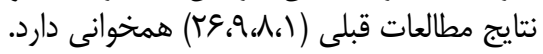
تجزيه خوشهاي

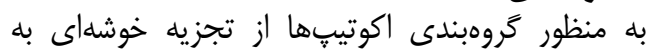

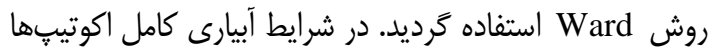

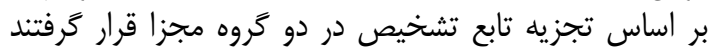

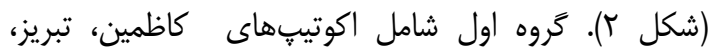

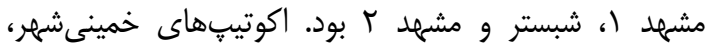

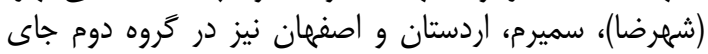

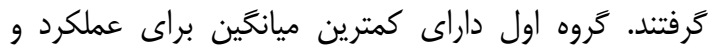

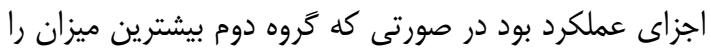

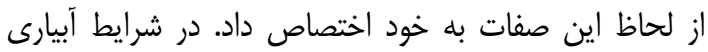

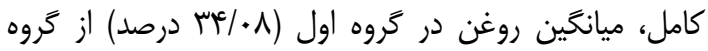

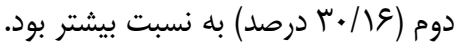




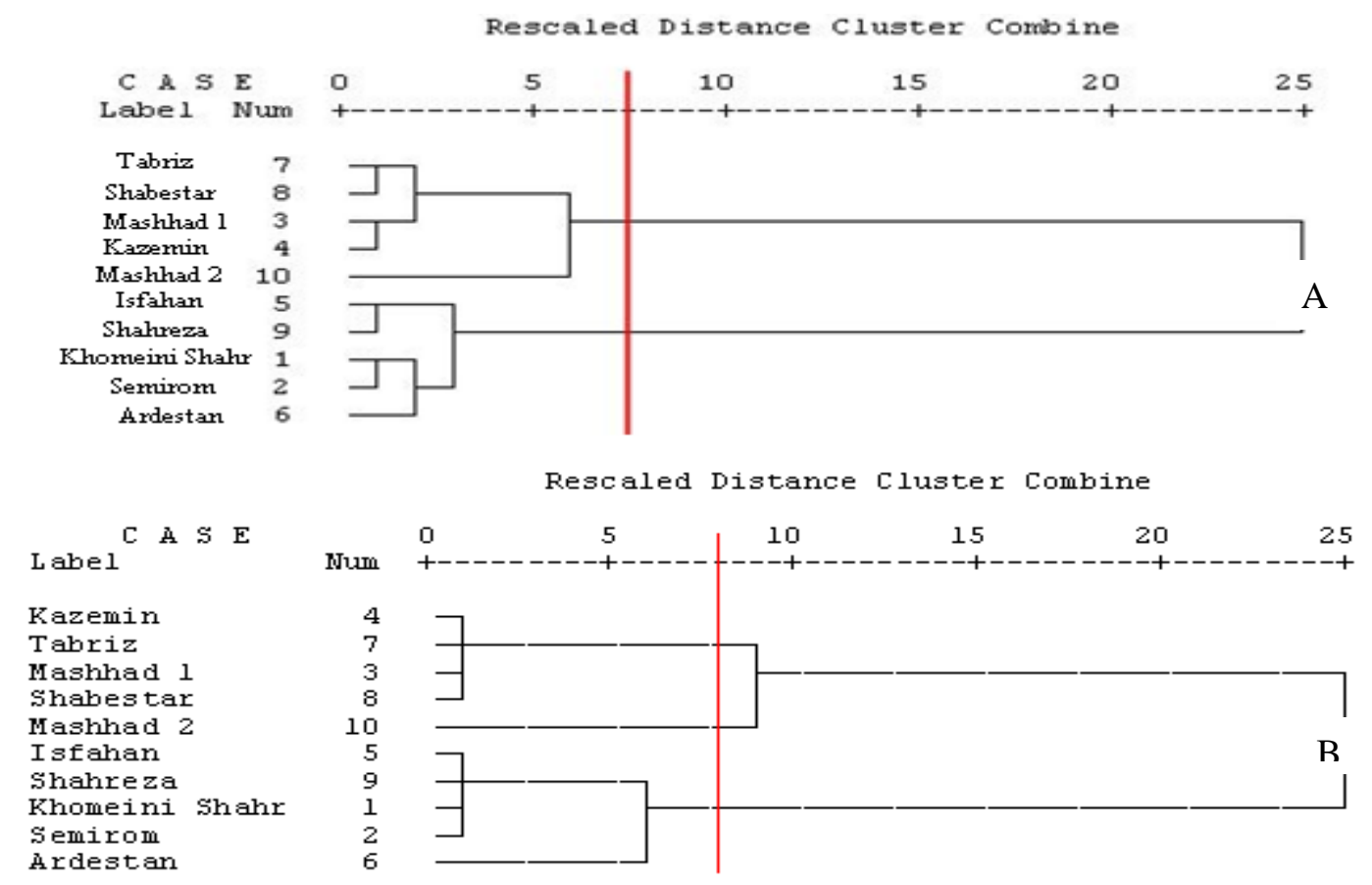

شكل r- دندروگرام حاصل از تجزيه خوشهاى اكوتيبهاى مختلف سياهدانه بر اساس صفات مورد مطالعه در شرايط آبيارى كامل (A) و تنش روش

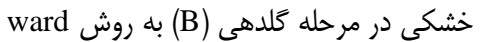

Figure 2. Dendrogram of different black cumin ecotypes based on the studied traits in full irrigation condition (A) and drought stress at flowering stage using ward's method
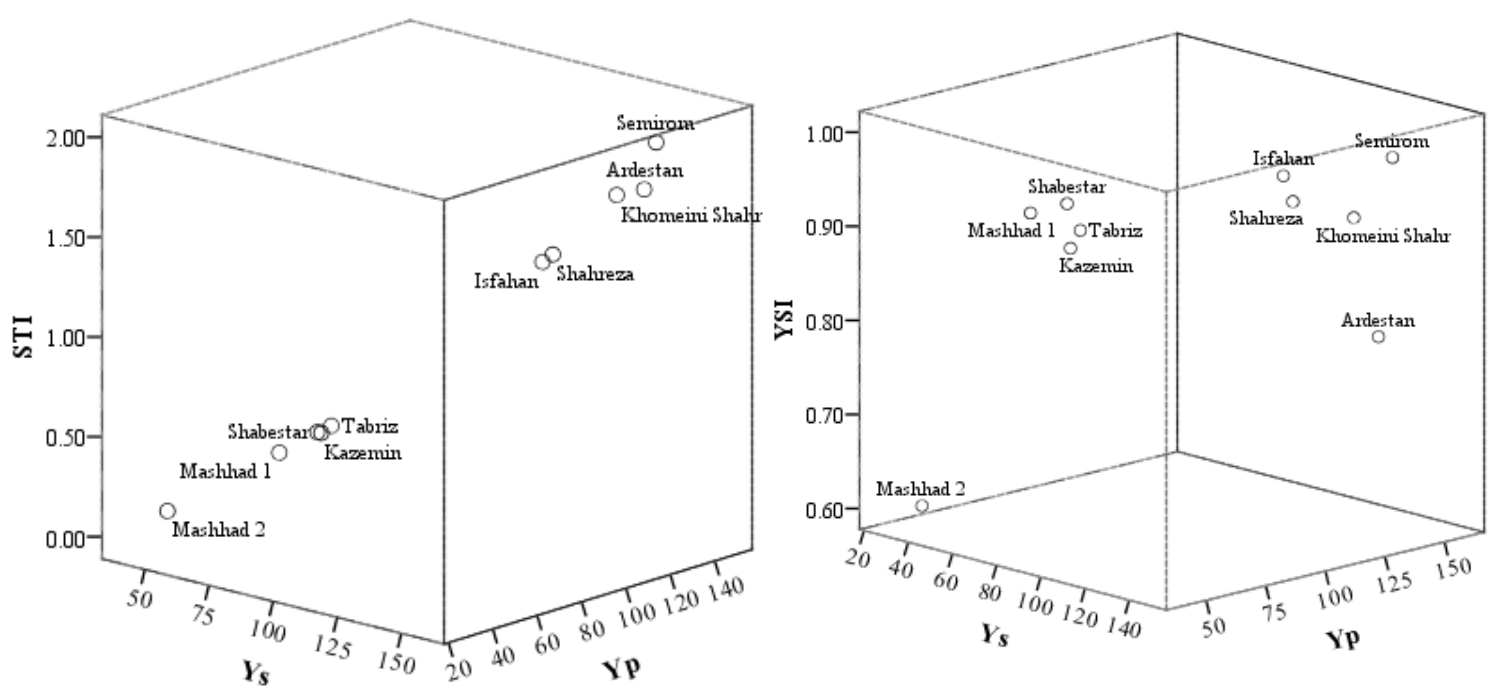

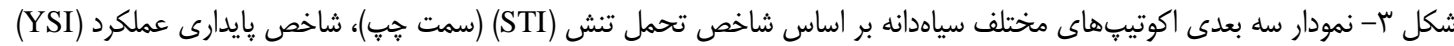

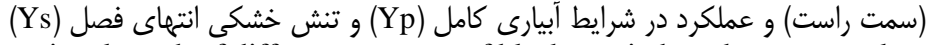

Figure 3. Three-dimensional graph of different ecotypes of black cumin based on stress tolerance index (STI) (left) yield stability index (YSI) (right) and yield under full irrigation (Yp) and end-season drought stress (Ys) conditions 


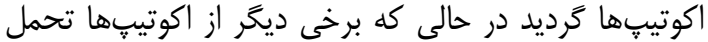

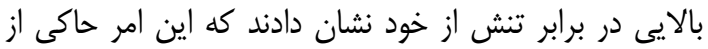

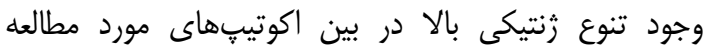

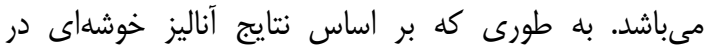

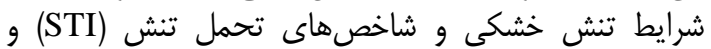

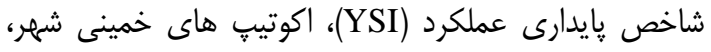

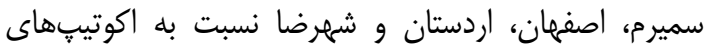

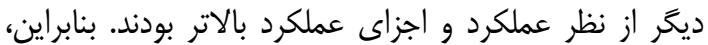

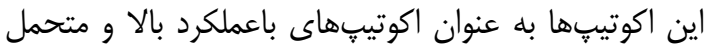
به ت تنش خشكى شناخته شدند.

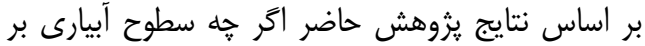

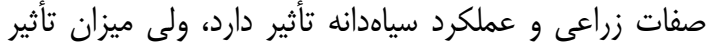

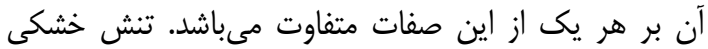

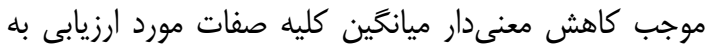

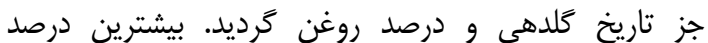

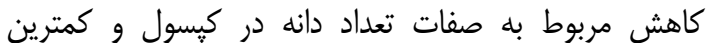

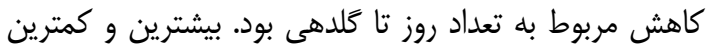

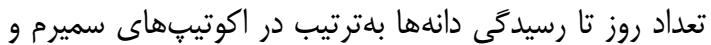

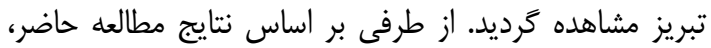

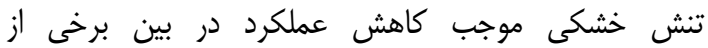

1. Akbarinia, A., M. Khosravifard, E. Sharifi Ashoorabadi and P. Babakhanlou. 2005. Effect of irrigaton intervals on yield and agronomic characteristics of black cumin (Nigella sativa). Iranian Journal of Medicinal and Aromatic Plants Research, 21(1): 65-73 (In Persian).

2. Bannayan, M., F. Najafi, M. Azizi, L. Tabrizi and M. Rastgoo. 2008. Yield and seed quality of Plantago ovata and Nigella sativa under different irrigation treatments. Industrial Crops and Products, 27(1): 11-16.

3. Beemarao Sankar, C.A., J. Paramasivam Manivannan, A. Kishore Kumar, R. Samasundaram and R. Panneerselvam. 2007. Drought induced biochemical modification and proline metabolism in Abelmoschus esculentus (L.) Moench. Acta Botanica Croatica, 66(1): 43-56.

4. Bohnert, H.J. and R.A. Bressan. 2001. Abiotic stresses, plant reactions, and new approaches towards understanding stress tolerance. Crop Science, 6: 81-100.

5. Bouslama, M. and W.T. Schapaugh. 1984. Stress tolerance in soybean. Part 1: evaluation of three screening techniques for heat and drought tolerance. Crop Science, 24(5): 933-937.

6. Cakir, R. 2004. Effect of water stress at different development stages on vegetative and reproductive growth of corn. Field Crop Research, 89(1): 1-16.

7. Chehl, N., G. Chipitsyna, Q. Gong and C. Yeo. 2009. Anti-inflammatory effects of Nigella sativa extract, thymoquinone, in pancreatic cancer cells. International Hepato-Pancreato-biliary Association Journal, 11(5): 373-381.

8. De Antuono, L.F., A. Moretti and A.F.S. Lovato. 2002. Seed yield, yield components, oil content and essential oil content and composition of Nigella sativa L. and Nigella damascena. Industrial Crops and Products, 15(1): 59-69.

9. Faravani, M., A.R. Razavi and M. Farsi. 2006. Study of variation in some agronomic and anatomic characters of Nigella sativa landraces in Khorasan. Iranian Journal of Medicinal and Aromatic Plants, 22(3): 193-197 (In Persian).

10. Farrokhinia, M., M. Roshdi, B. Pasban Eslam and S. Rezadoost. 2009. Study of effects drought stress on the yield and som of the vegetative characters in safflower. Journal of Research in Crop Science, 2(5): 1-11 (In Persian).

11. Fernandez, G.C.J. 1992. Effective selection criteria for assessing stress tolerance. In: Kuo C.G. (Ed.). Proceedings of the international symposium on adaptation of vegetables and other food crops in temperature and water stress. Publication, Tainan, Taiwan.

12. Fischer, R.A. and R. Maurer. 1978. Drought resistance in spring wheat cultivars I. Grain yield responses. Crop and Pasture Science, 29(5): 897-912.

13. Ghamarnia, H., H. Khosravy and S. Sepehri. 2010. Yield and water use efficiency of (Nigella sativa L.) under different irrigation treatments in a semi-arid region in the west of Iran. Journal of Medicinal Plants Research, 4(16): 1612-1616 (In Persian).

14. Haj Seyed Hadi, M.R., M.T. Darzi and G. Riazi. 2016. Black cumin (Nigella sativa L.) yield affected by irrigation and plant growth promoting bacteria. Journal of Medicinal Plants and By-products, 5(2): 125-133 (In Persian).

15. Hassani, A. 2006. Effect of water deficit stress on growth, yield and essential oil content of Dracocephalum moldavica. Iranian Journal of Medicinal and Aromatic Plants Research, 22(3): 256261 (In Persian).

16.Iqbal, M.S., A. Ghafoor, F.M. Abbasi, A.S. Qureshi and H. Ahmad. 2011. Study of nutritional characteristics, mineral nutrients and agro-biodiversity in black cumin (Nigella sativa L.) genotypes from Pakistan. African Journal of Biotechnology, 10(66): 14757-14766.

17. Ismail, M.Y.M. 2009. Therapeutic role of prophetic medicine habbat el baraka (Nigella sativa L.)-A review. World Applied Sciences Journal, 7(9): 1203-1208. 
199 ثرُوهشنامه اصلاح كياهان زراعى/ سال دوازدهم/ شماره عب/ تابستان

18. Jaleel, C.A., P. Manivannan, A. Wahid, M. Farooq, R. Somasundaram and R. Panneerselvam. 2009. Drought stress in plants: a review on morphological characteristics and pigments composition. International Journal Agriculture Biology, 11(1): 100-105.

19. Kafy, M. and M. Rostami. 2009. Yield characteristics and oil content of three safflower. Iranian Journal of Field Crops Research, 5(1): 121-132 (In Persian).

20. Khalid, K.A. 2006. Influence of water stress on growth, essential oil and chemical composition of herbs (Ocimum sp.). International Agrophysics, 20(4): 289-296.

21.Leport, L., N.C. Turner, R.J. French. D. Tennant, B.D. Thomson and K.H.M. Siddique. 1998. Water relation, gas exchange, and growth of cool-season grain legumes in a Mediterranean-type environment. European Journal of Agronomy, 9(4): 295-303.

22. Monivannan, P., C. Abdul Jaleel, B. Sankal, A. Kishore Kumar, R. Sornasundaram, G.M.A. Lakshmanan and K. Panneerselvam. 2007. Growth, biochemical modification and proline metabolism in (Helianthus annus L.) as induced by drought stress. Colloids and Surfaces, 59(2): 141-149.

23. Mozzafari, F., S. Ghorbanli, M. Babai and A. Farzami. 2000. The effect of water stress on the seed oil of Nigella sativa L. Journal of Essential Oil Research, 12(1): 36-38.

24. Nickavar, B., F. Mojab, K. Javidnia and M.A. Amoli. 2003. Chemical composition of the fixed and volatile oils of (Nigella sativa L.) from Iran. Zeitschrift für Naturforschung C. A Journal of Biosciences, 58(9-10): 629-631.

25. Omar, A., S. Ghosheh, A. Abdulghani, A. Houdi and P.A. Crookscor. 1999. High performance liquid chromatographic analysis of the pharmacologically active quinones and related compounds in the oil of the black seed (Nigella sativa). Journal of Pharmaceutical and Biomedical Analysis, 19(5): 757762.

26. Rezvan Beidokhti, S., S. Sanjani, A. Dashtban and I. Hesam Arefy. 2012. Evaluation of yield and yield components of black cumin (Nigella sativa L.) under different plant density and limited irrigation condition. Iranian Journal of Field Crops Research, 10(2): 382-391 (In Persian).

27. Rosielle, A.A. and J. Hanbilin. 1981. Theoretical aspects of selection for yield in stress and non-stress environments. Crop Science, 21(6): 943-946.

28. Salehi, I. and M.H. Surmaghi. 2008. Nigella Sativa. Herbal Medicine and Herbal Therapy, Donyay Taghziah press, 76(2): 216 -229 (In Persian).

29. Shahattary, F.S. and C. Mansourifar. 2017. The effect of drought stress on morphological and physiological traits and essence percentage of medicinal plant, Nigella sativa. Bioscience Biotechnology Research Commnications, 1: 298-305.

30. Soleimani A, M. Valizadeh, R. Darvishzadeh, S. Aharizad and H. Alipour. 2017. Evaluation of yield and yield component of spring barely genotypes under late season drought stress. Journal of Crop Breeding, 9(23): 105-116 (In Persian).

31. Taiz, J. and E. Zeiger. 2001. Plant Physiology. 3rd ed. Sunderland, Sinauer Associates. 379 pp.

32. Vafabakhsh, J., M., Nasiri Mahlati A.R. Kocheki and M. Azizi 2009. Effects of water deficit on water use efficiency and yield of canola cultivars (Brassica napus L.). Iranian Journal of Field Crops Research, 7(1): 297-304 (In Persian).

33. Zali, H., T. Hasanloo, O. Sofalian, A. Asghari and M. Zeinalabedini. 2016. Drought stress effect on physiological parameter and amino acids accumulations in canola. Journal of Crop Breeding, 8 (18):191-203 (In Persian). 


\title{
Evaluation of Black Cumin (Nigella sativa L.) Ecotypes under Drought Stress Conditions at Flowering Stage
}
Hassan Sardari ${ }^{1}$, Rasool Asghari Zakaria ${ }^{2}$, Nasser Zare ${ }^{3}$, Lila Ghafarzadeh Namazi ${ }^{4}$ and Mina Moghaddaszadeh ${ }^{5}$
1, 3 and 5- Graduted M.Sc. Student, Associate Professor and Graduted PhD. Student of Plant Breeding, University of Mohaghegh Ardabili
2- Professor, Faculty of Agriculture and Natural Resources, University of Mohaghegh Ardabili, Ardabil, I.R. Iran, (Corresponding author: r-asghari@uma.ac.ir)
4- Assistant Professor, Meshkin Shahr Faculty of Agriculture, University of Mohaghegh Ardabili, Ardabil, Iran \\ Recived: December 2, 2019 \\ Accepted: February 29, 2020
}

\begin{abstract}
Water deficiency causes crop yield loss, especially in flowering and pollination stage. This investigation was conducted out as a field experiment in Agriculture Faculty of University of Mohaghegh Ardabili in 2018, to evaluate drought tolerance of ecotypes at flowering stage. All plots were arranged in split plots based on a randomized complete block design with three replications. The main factor of experiment included two irrigation levels (full irrigation and irrigation until flowering stage) and sub-factor consisted of 10 different ecotypes of black cumin. Analysis of variance showed that the effect of drought stress on all studied traits except flowering date was significant. Drought stress significantly reduced the mean of all traits except flowering date and oil percentage. Comparison of mean data showed that the highest grain yield and oil content were observed in Semirom and Kazemain ecotypes, respectively. Also, the highest 1000-grain weight was obtained and in Ardestan and Khomeini Shahr ecotypes at full irrigation. Based on the results of cluster analysis under drought stress condition, the studied ecotypes were divided into three groups. Ecotypes of third group including Khomeini Shahr, Semirom, Isfahan, Ardestan and Shahreza had higher yield and yield components than ecotypes in other groups. Therefore, these ecotypes were identified as high yielding and drought tolerant. Also, three-dimensional plots based on stress tolerance index (STI), yield stability index (YSI), yield under full irrigation (Yp) and end-season drought stress (Ys) showed that the ecotypes of Khomeini Shahr, Semirom, Isfahan, Shahreza and Ardestan are considered as superior ecotypes in both conditions.
\end{abstract}

Keywords: Cluster analysis, Drought stress, Stress tolerance index, Black cumin, Seed yield 